\title{
EDITORIAL
}

\section{Neglected Diseases: Burden and attention}

\author{
Ahmed Ali
}

Neglected diseases are largely infectious diseases that have burdened humanity for centuries, but currently receiving little attention. Infectious diseases in their long histories have resulted in considerable morbidities, disabilities and deformities, often subjecting to stigma. The magnitude of their impact on health and labor productivity led to a thorough understanding about their biology, and epidemiology, and effective control tools and strategies were developed for most of them. Owing to the overall socio-economic development and improved living conditions, opportunities for transmission of communicable diseases are reduced in the developed parts of the world and in limited parts of the metropolitan areas of developing countries. Neglected diseases are concentrated almost exclusively in impoverished populations living in areas where provision for basic environmental health, including vector control and minimum package of health service are strictly limited.

Neglected diseases share features that allow them to flourish under circumstances of poverty and utter ignorance, where they cluster and often overlap in distribution. Lack of adequate and safe water and poor sanitation sustain transmission cycles and favor the proliferation of vectors. Restricted access to health services, low levels of health consciousness, insufficient nutrition and compromised personal hygiene increase vulnerability to infection and hinder efforts for prevention. Curative interventions, if any, are sought late to prevent permanent impairments and the inevitable death. Mobility facilitated by modern transport and frequently precipitated by natural and man made disasters as well contributes to enhance the problem.

The consequences from neglected diseases include school absenteeism, compromised growth and development, loss of labor productivity, heavy burden on home-based care, inefficient land use and food insecurity among others. The leading causes of death in developing countries are infectious diseases, which are preventable and largely curable. Women and children are the most vulnerable and the principal victims of the ailments. It is of a paramount importance to recognize these factors in order to develop promotive, preventive and early curative strategies to limit their substantial impact.

Neglect for diseases of public health importance could occur at different levels. Owing to paucity of information, diseases could be often hidden- out of sight, poorly documented and silent, as those most affected could be inarticulate for various reasons. As a consequence, diseases could rarely be given priority to get their respective share of whatever meager resources. Neglected diseases could as well lack visibility at an international level since they are specific to certain geographical and environmental conditions and not perceived as direct threats to developed countries. The other cause for reluctance for intervention could be their low mortality, in spite of their considerable impairment or permanent disability.

Although infectious diseases remain to be significant causes of morbidity and mortality and a sizeable of them are aptly designated as neglected, I am of firm conviction that prevalent chronic noninfectious diseases are not as well accorded with due recognition and attention, at least in our country. This illustrates the huge information gap to be bridged by research and need for improvement of the management information system, to ease policy and decision making and facilitate implementation at facility level. Instituting and strengthening surveillance and reporting mechanisms, sponsoring multidisciplinary research and incorporating monitoring and evaluation procedures in to the system will ensure sustainable intervention to prevent and control neglected diseases, irrespective of their genesis. 